\title{
Surgical versus conservative treatment for high-risk stress fractures of the lower leg (anterior tibial cortex, navicular and fifth metatarsal base): a systematic review
}

\author{
Wouter H Mallee, ${ }^{1}$ Hanneke Weel, ${ }_{1}^{1}$ C Niek van Dijk, ${ }^{1}$ Maurits W van Tulder, ${ }^{2}$ \\ Gino M Kerkhoffs, ${ }^{1}$ Chung-Wei Christine Lin ${ }^{3}$
}

\begin{abstract}
- Additional material is published online only. To view please visit the journal online (http://dx.doi.org/10.1136/ bjsports-2013-093246).

${ }^{1}$ Department of Orthopaedic Surgery, Academic Medical Center of Amsterdam, Amsterdam, The Netherlands ${ }^{2}$ Department Health Sciences, EMGO+ Institute for Health and Care Research, Faculty of Earth and Life Sciences, VU University Amsterdam, Amsterdam, The Netherlands ${ }^{3}$ The George Institute for Global Health, Sydney Medical School, The University of Sydney, Sydney, Australia
\end{abstract}

Correspondence to Dr Wouter H Mallee, Academic Medical Center of Amsterdam, Department of Orthopaedic Surgery, Secretariaat G4-Noord, Meibergdreef 9 , Amsterdam Z-0 1105 AZ, The Netherlands; w.h.mallee@amc.uva.nl

Accepted 22 July 2014 Published Online First 19 August 2014

\section{ABSTRACT}

Aim To compare surgical and conservative treatment for high-risk stress fractures of the anterior tibial cortex, navicular and proximal fifth metatarsal.

Methods Systematic searches of CENTRAL, MEDLINE, EMBASE, CINAHL, SPORTDiscus and PEDro were performed to identify relevant prospective and retrospective studies. Two reviewers independently extracted data and assessed methodological quality. Main outcomes were return to sport and complication rate.

Results 18 studies were included ( 2 anterior tibia $(\mathrm{N}=31), 8$ navicular $(\mathrm{N}=200)$ and 8 fifth metatarsal $(\mathrm{N}=246))$. For anterior tibial fracture, no studies on initial surgery were eligible. Conservative treatment resulted in high complication rates and few cases returned to sport. For navicular fracture, a weighted mean return to sport of 22 for conservative and 16 weeks for surgical treatment was found. Six weeks of non-weightbearing cast was mostly used as conservative treatment. Surgical procedures varied widely. For the fifth metatarsal fracture, weighted mean return to sport was 19 for conservative and 14 weeks for surgical treatment. Surgery consisted of intramedullary screw fixation or tension band wiring. For conservative methods, insufficient details were reported. Overall, there was a high risk of bias; sample sizes were small and GRADE level of evidence was low.

Conclusions Strong conclusions for surgical or conservative therapy for these high-risk stress fractures cannot be drawn; quality of evidence is low and subjected to a high risk of bias. However, there are unsatisfying outcomes of conservative therapy in the anterior tibia. The role of initial surgery is unknown. For the navicular, surgery provided an earlier return to sport; and when treated conservatively, weightbearing should be avoided. For the fifth metatarsal, surgery provided the best results. Treatment decision-making would greatly benefit from further prospective research.

Study registration number PROSPERO database of systematic reviews: CRD42013004201.

\section{CrossMark}

To cite: Mallee $\mathrm{WH}$ Weel $\mathrm{H}$, van Dijk $\mathrm{CN}$, et al. $\mathrm{Br} J$ Sports Med

2015;49:370-376

\section{INTRODUCTION}

The time to return to sport or full activity is an important issue in the treatment of stress fractures as they mainly occur in (high-level) athletes or military personnel. ${ }^{1}{ }^{2}$ These injuries usually appear in the lower leg due to repetitive loading and highimpact forces applied during training and matches. ${ }^{3}$
Notorious locations in this type of injury are the anterior tibial cortex, navicular bone and proximal fifth metatarsal. ${ }^{4}$ Owing to hypovascularity or a watershed area, ${ }^{5-9}$ these stress fractures are prone to delayed union or non-union. Complication rates are high and patients are often absent from duty or sport for a long time, sometimes resulting in the end of a career. Therefore, these fractures are known as 'high-risk' injuries. ${ }^{10-12}$ Clinical decisionmaking and adequate treatment of this demanding group of patients is crucial to facilitate a return to their normal level of activity.

\section{Anterior tibial cortex}

Of all tibial stress fractures, the anterior cortex is injured in only $5-15 \%,{ }^{13}{ }^{14}$ but of the three highrisk stress fractures, these might create the biggest threat for an athletic or military career. They differ from the more common and easier to treat posteromedial tibial stress fractures, as the anterior part is the tension side of the tibia. Compression of the fracture site is therefore hindered. From start of treatment until complete healing can take up to 20 months. ${ }^{15} 16$ Owing to its location and low prevalence, symptoms are usually not recognised as stress fracture. This can result in a big delay in definitive diagnosis and initiation of treatment.

\section{Navicular}

Navicular stress fractures are one of the more common stress fractures, ${ }^{17}$ especially in runners and basketball players. ${ }^{18}$ These stress fractures can present as partial or complete fractures. In type I, a fracture of the dorsal navicular cortex is present, type II fractures disseminate into the navicular body and type III fractures extend to a second cortex of the navicular. Severity of the fracture is believed to correlate with the outcome. ${ }^{19}$

\section{Proximal fifth metatarsal}

The proximal fifth metatarsal stress fracture is mainly seen in soccer, football and basketball players. ${ }^{4} 2021$ Several theories about risk factors like cavovarus foot ${ }^{22} 23$ and curved type fifth metatarsal bone in the medial oblique radiograph ${ }^{23}$ have been reported. Different prognostic factors like Torg classification, ${ }^{24}$ zones according to Lawrence $^{25}$ and the plantar gap ${ }^{26}$ might predict outcome and guide treatment. A plantar gap of more than $1 \mathrm{~mm}$ or Torg 2 or 3 fractures are likely to have the most prolonged healing time. ${ }^{26}$ 
Whereas the impact of these three lower leg stress fractures can be severe in this active population, identifying the best treatment options that allow an early return to sport or activity is much needed. However, it remains unclear which option is most effective. ${ }^{27} 28$ The purpose of this systematic review is to compare surgical and conservative treatment for high-risk stress fractures of the lower leg on return to activity, work and sport.

\section{METHODS}

The protocol for this study was registered in the PROSPERO database (CRD42013004201). The review is reported according to the 2009 PRISMA Checklist. ${ }^{29}$

\section{Search strategy}

The following electronic databases were systematically searched: Central via Cochrane Library, MEDLINE via PubMed, EMBASE, CINAHL and SPORTDiscus via EBSCO-host from inception to 26 March 2013 (see online supplementary appendix 1) and PEDro from inception to 9 April 2013. The general search terms were 'tibia', 'navicular' and 'metatarsal' to identify the right location. For the right condition, we used 'stress fracture' and 'fatigue fracture'. 'Conservative treatment' and 'surgery' were added to look for treatment studies and their outcome with 'treatment outcome', 'return to sports' and 'fracture healing'. The reference lists of relevant reviews and included studies were screened for additional articles.

\section{Selection criteria}

Because of the expected low number of randomised controlled trials (RCTs), other study designs such as non-randomised trials and prospective cohorts with or without a comparison were included as well. Case series were included only if the sample was consecutively collected and number of patients was at least 10. No time or language restriction was applied. Male and female samples of all age groups, athletes or non-athletes, with stress fractures to the anterior tibial cortex, navicular bone or proximal fifth metatarsal undergoing surgical or conservative treatments (including rest) were included. The primary outcome was time to return to activity, work and sport. Secondary outcomes were time to healing (clinical and radiological), complication rate (eg, refractures, non-unions, postoperative infections) and economic data.

\section{Data extraction}

Study selection and data extraction were performed by two review authors (WHM and HW), independently. Disagreements were discussed in a consensus meeting and a third reviewer (C-WCL) made a final decision if disagreements persisted.

Titles and abstracts were screened for eligibility. After initial selection, full article review was performed and a reason for exclusion provided at this stage. The following data were extracted from included studies: study design, sample source and size, participant characteristics, time from onset of symptoms to diagnosis and start of treatment, type of treatment, timing of follow-up assessments and data on the primary and secondary outcomes. Data were extracted for all follow-ups available in each included study. The full text screening form and the data extraction form were specially created for the review and were piloted before use.

\section{Strategy for data synthesis}

Data were presented per fracture location (anterior tibial cortex/ navicular/proximal fifth metatarsal), by type of treatment (surgical or conservative) and, if available, by the type of comparator.
Time units of the outcomes were converted (if needed) and presented in weeks, other time units were converted and presented in months. If enough studies were present that were clinically and statistically homogeneous and data on them adequately reported, a meta-analysis was performed using a fixed-effects model. In case of statistical heterogeneity, a random-effects model was used. If meta-analysis was not possible, results were reported in a descriptive manner. The need to explore for statistical heterogeneity was determined by a combination of visual inspection of the results (eyeball test) and an $\mathrm{I}^{2}$ greater than $40 \%$.

For all continuous outcomes, a standardised mean difference was calculated when studies used different methods to measure the same outcome, or weighted mean difference and SDs if the same methods were used. Between-group differences were reported with 95\% CIs. For dichotomous data, risk ratios and 95\% CIs were calculated. Categorical data were presented in a descriptive manner.

\section{Risk of bias assessment}

Two review authors performed the risk of bias (RoB) assessment (WHM and HW) using the Cochrane collaboration Risk of Bias tool. Disagreements were discussed in a consensus meeting and a third reviewer (C-WCL) made a final decision if disagreements persisted. The following items were assessed: random sequence generation, allocation concealment, blinding of outcome assessment, incomplete outcome data, selective outcome reporting and other sources of bias. The items were scored: low risk, high risk or unclear risk according to the descriptions in the latest version of the Cochrane handbook. ${ }^{30}$

The overall quality of the evidence and strength of recommendations was evaluated using the GRADE approach. ${ }^{31} 32$ The quality of the evidence for a specific outcome is based on five principal domains: (1) limitations (due to, eg, RoB), (2) consistency of results, (3) directness (ie, generalisability), (4) precision (sufficient data with narrow CIs) and (5) other limitations (eg, publication bias). To improve the readability of this review, GRADE tables were to be generated only when two or more studies were to be analysed for a given comparison. Single studies are considered to provide 'low' or 'very low-quality evidence', depending on whether they are associated with a low or high RoB, respectively. In addition, GRADE tables were generated for the primary outcome measures only. The following levels of the quality of the evidence were applied:

High quality: Further research is very unlikely to change the level of evidence. There are sufficient data with narrow CIs. There are no known or suspected reporting biases.

Moderate quality: Further research is likely to have an important impact on confidence in the estimate of effect and may change the estimate; one of the domains is not met.

Low quality: Further research is very likely to have an important impact on confidence in the estimate of effect and is likely to change it; two of the domains are not met.

Very low quality: Great uncertainty about the estimate; three of the domains are not met.

\section{RESULTS}

The systematic search yielded a total of 927 hits resulting in 540 unique references for screening (figure 1). During full article review of the 93 articles that met the inclusion criteria following title and abstract screening, most articles were excluded because the number of cases was lower than 10, or the fracture was not caused by repetitive stress. Eighteen small studies, 16 retrospective case series and 2 prospective cohorts, were appropriate for 


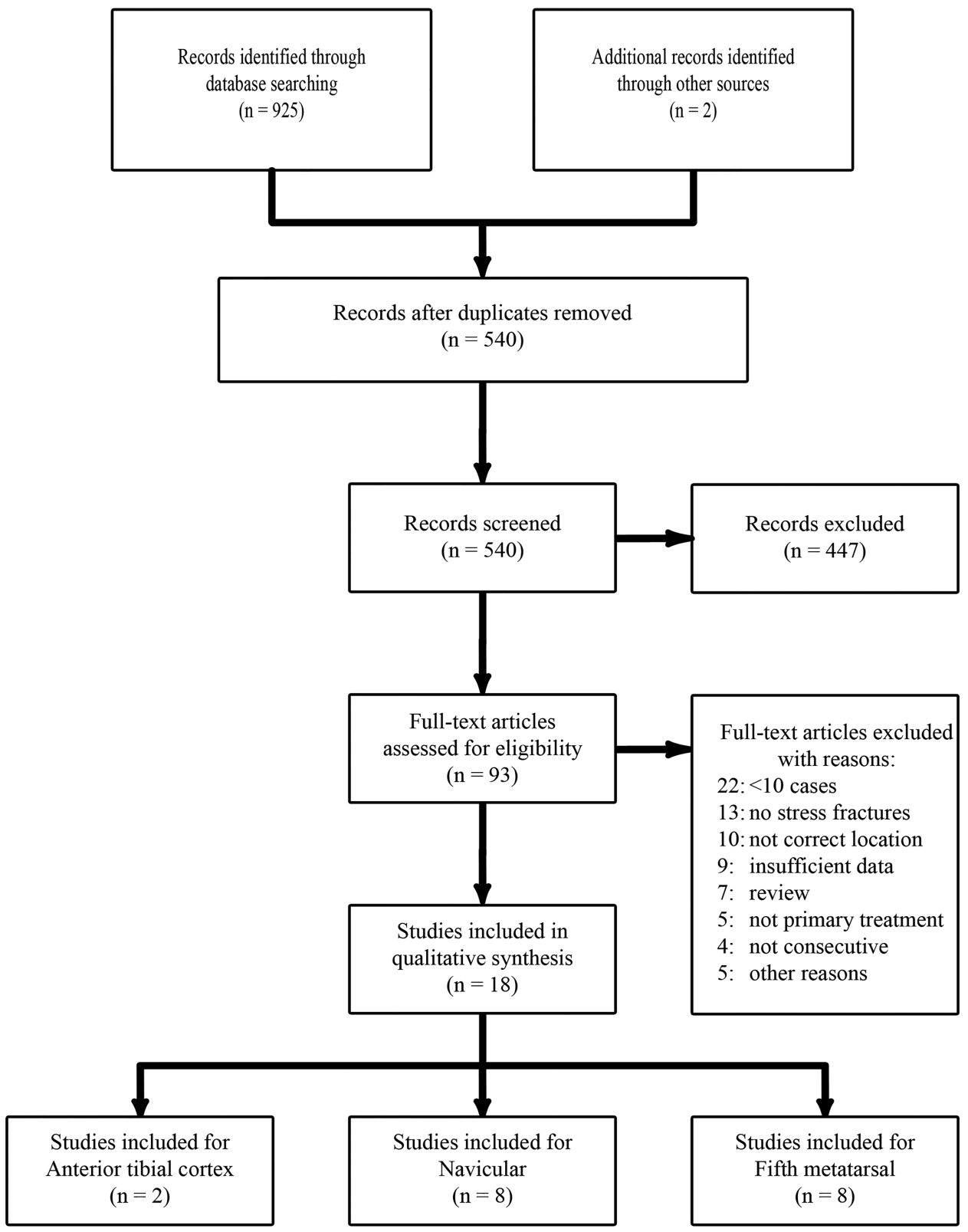

Figure 1 PRISMA flow diagram.

inclusion in this review: 2 for the anterior tibial cortex, 8 for the tarsal navicular and 8 for the fifth metatarsal. The characteristics per study are presented in online supplementary table S1.

\section{Risk of bias assessment}

All studies had a high risk of selection bias since there were no RCTs identified (table 1). In addition, risk of detection bias was high when there was no blinding of the outcome assessor ${ }^{6} 19$ 33-36 or unclear when blinding was not reported. ${ }^{15} 2021 \quad 37-41$ Some studies were clear and complete in their data reporting, resulting in a low risk to attrition and reporting bias. Risk of other biases was high, because most studies used a retrospective design.

\section{Anterior tibial cortex}

No studies comparing surgical and conservative treatment were found (table 2). Two small retrospective case series $(n=14$ and 17) were included, resulting in very low-quality evidence. ${ }^{15} 39$ Patients were all recreational or elite level athletes, aged between 11-44 years old. After a delay from onset of symptoms until treatment of up to 36 months, all patients were initially treated with rest from sports and/or immobilisation (method unspecified). Johansson $e t a l^{15}$ reported a return to sport rate of $55 \%$. Orava et $\mathrm{al}^{39}$ found a time to return to sport of 24 weeks (range 12-40) for the healed fractures but the non-unions returned to sport 28.4 weeks (16-64) after subsequent surgery. In total, $13 / 14(93 \%)^{15}$ and $9 / 17(53 \%)^{39}$ anterior tibial stress fractures did not unite after initial conservative treatment. Of these patients, 13 needed secondary surgery consisting of transverse drilling and excision of the granulated tissue and in some cases autologous bone graft was added. No studies were identified that reported on initial surgical treatment.

\section{Navicular}

No RCTs were identified (table 2). A total of eight studies, seven retrospective series and one prospective study, were found, describing 200 fractures. Only three patients were non- 
Table 1 Cochrane Risk of Bias assessment-web appendix

\begin{tabular}{|c|c|c|c|c|c|c|}
\hline \multirow[b]{2}{*}{ Study } & \multicolumn{2}{|l|}{ Selection bias } & \multirow{2}{*}{$\begin{array}{l}\text { Detection bias } \\
\text { Blinding of outcome } \\
\text { assessors }\end{array}$} & \multirow{2}{*}{$\begin{array}{l}\text { Attrition bias } \\
\text { Incomplete outcome } \\
\text { data }\end{array}$} & \multirow{2}{*}{$\begin{array}{l}\text { Reporting bias } \\
\text { Selective outcome } \\
\text { reporting }\end{array}$} & \multirow[b]{2}{*}{ Other bias } \\
\hline & $\begin{array}{l}\text { Random sequence } \\
\text { generation }\end{array}$ & $\begin{array}{l}\text { Allocation } \\
\text { concealment }\end{array}$ & & & & \\
\hline \multicolumn{7}{|l|}{ Anterior tibia } \\
\hline Johansson et al & - & - & $?$ & + & $?$ & - \\
\hline Orava et al & - & - & $?$ & - & $?$ & - \\
\hline \multicolumn{7}{|l|}{ Navicular } \\
\hline Benazzo et al & - & - & - & - & - & - \\
\hline Bojanic et al & - & - & - & + & + & + \\
\hline Burne et al & - & - & $?$ & + & $?$ & - \\
\hline Khan et al & - & - & $?$ & - & $?$ & - \\
\hline McCormick et al & - & - & - & + & - & - \\
\hline Saxena et $a l^{19}$ & - & - & - & - & - & - \\
\hline Saxena and Fullem ${ }^{36}$ & - & - & - & - & - & - \\
\hline Torg et al & - & - & - & + & - & - \\
\hline \multicolumn{7}{|l|}{ Fifth metatarsal } \\
\hline Chuckpaiwong et al & - & - & $?$ & - & - & - \\
\hline DeLee et al & - & - & $?$ & + & $?$ & - \\
\hline Ekstrand et al & - & - & $?$ & + & + & + \\
\hline Josefsson et al & - & - & $?$ & + & - & - \\
\hline Lee et al & - & - & $?$ & $?$ & $?$ & - \\
\hline Nagao et al & - & - & $?$ & + & $?$ & - \\
\hline Pecina et al & - & - & - & + & - & - \\
\hline Popovic et al & - & - & - & + & - & - \\
\hline
\end{tabular}

athletes, the rest were athletes of recreational or elite level. Their median age was in the mid-20s. The mean time from onset of pain until treatment was 2 months and ranged from immediate treatment to 5 years' delay. The received conservative and surgical treatments varied widely among studies and the data reported on return to sport and time until healing were skewed, so it was not possible to perform a meta-analysis.

Four retrospective case series included surgically and conservatively treated patients. ${ }^{6} 1936 \quad 38$ Two studies reported an earlier return to sport after surgical treatment compared with conservative treatment. ${ }^{19} 36$ In one study, this difference was statistically significant (average: 12.4 vs 17.2 weeks), but with great dispersion (range: $6-52$ weeks). ${ }^{19}$ A second study by the same authors showed no significant differences between the two treatments ( 14.8 vs 16.4 weeks). ${ }^{36}$ The weighted mean time to return to sport was 16.4 weeks in the surgically treated patients $^{6} 193638$ and 21.7 weeks in patients treated conservatively with non-weightbearing cast (NWBC) >6 weeks. ${ }^{6} 19343638$ Khan reported no difference in successfully return to sport within 12 months between conservative and surgically treated patients $(86 \%$ vs $83 \%$, respectively). The success rates of NWBC $<6$ weeks and weightbearing (WB) treatment were lower than $50 \% .{ }^{38}$ More severely classified fractures that extend to the opposite cortex (table 3) may prolong the time to return to sport, ${ }^{19} 36$ but a diagnostic delay did not. ${ }^{19}$

Two retrospective case series reported on the effects of conservative treatment only. Benazzo et $a l^{33}$ evaluated the application of electrostimulation $(n=13)$ and reported an average time to clinical healing of 8.6 weeks (range 4-10.7). Burne et al reported an average time to clinical healing of 4 years (range 1.1-15.3). None of the other included studies provided data on time to healing.

Adverse events were generally poorly reported (low-quality reporting). They were reported in five studies. Of the 35 surgically treated patients, complications included two hardware removals, one non-union and one superficial infection treated with oral antibiotics. In the 165 conservatively treated patients, 39 treatment failures consisted of 32 in the WB group, ${ }^{38}$ as well as two non-unions and three delayed unions in the WB group. This WB group also had 13 refractures. $^{6} 193437$ There was an $80 \%$ treatment success among the patients treated with NWBC and a $29 \%$ success among the patients treated with some form of WB rest or immobilisation. ${ }^{38}$

\section{Proximal fifth metatarsal}

A total of 246 cases from eight studies were eligible for inclusion (table 2). The athletic level of the participants was mainly elite and their age ranged between 13 and 45 years. One study looked at conservative treatment, ${ }^{41}$ five studied surgical treatment $^{20} 26{ }^{42-44}$ and two studied both. ${ }^{21} 40$ Different types of conservative and surgical treatments were administered. No RCTs were identified, one study used a prospective design ${ }^{21}$ and all other studies were retrospective case series.

Of the two studies that included surgically and conservatively treated patients, Chuckpaiwong et al reported a significant quicker return to sport for surgical treatment (mean 15.2, SD 10.5 weeks) compared with conservative treatment (mean 26.3, SD 11.0 weeks). In addition, one out of the eight conservatively treated patients required secondary surgery due to non-union of the fracture. The other study, a prospective cohort by Ekstrand et al, reported an earlier return to sport for the conservative group of 9.9 vs 12 weeks for the surgical group, but the range was wide and this difference was not statistically significant. Of the 6 patients treated conservatively, three refractures and one non-union occurred while 5 of the 14 patients treated surgically had a refracture and no non-unions occurred. When all eight studies were pooled together, the weighted mean time to return to sport was 13.8 (SD 2.8) weeks in the surgically treated patients and 19.2 (SD 6.3) weeks in the conservatively treated patients. 


\begin{tabular}{|c|c|c|c|c|c|c|c|c|c|c|}
\hline \multirow[b]{2}{*}{ Reference } & \multirow[b]{2}{*}{ Treatment } & \multirow[b]{2}{*}{$\begin{array}{l}\text { Onset until treatment } \\
\text { Month (range) }\end{array}$} & \multicolumn{3}{|c|}{ Primary outcomes } & \multicolumn{2}{|c|}{ Secondary outcomes } & \multicolumn{3}{|c|}{ Complications } \\
\hline & & & $\begin{array}{l}\text { RT sport } \\
\text { Weeks (range) }\end{array}$ & $\begin{array}{l}\text { RT sctivity } \\
\text { Weeks (range) }\end{array}$ & $\begin{array}{l}\text { RT work } \\
\text { Weeks (SD) }\end{array}$ & $\begin{array}{l}\text { Clinical healing } \\
\text { Weeks (range) }\end{array}$ & $\begin{array}{l}\text { Radiological healing } \\
\text { Weeks (range) }\end{array}$ & $\begin{array}{l}\text { Non-unions } \\
\text { (delayed) }\end{array}$ & Refractures & Postoperative \\
\hline \multicolumn{11}{|l|}{ Anterior tibial cortex } \\
\hline Johansson et al & C & $14.8(1-36)$ & $n=6$ & $x x$ & $x x$ & $x x$ & $\mathrm{xx}$ & 13 & $x x$ & $x x$ \\
\hline Orava et al & C & $7(3-24)$ & $24(12-40)$ & $x x$ & $x x$ & $x x$ & is RT sports & 9 & 1 & $x x$ \\
\hline \multicolumn{11}{|l|}{ Navicular } \\
\hline Benazzo et al & C & 7.5 & All & $x x$ & $x x$ & $8.6(4-10.7)$ & is Clinical healing & 0 & 0 & $x x$ \\
\hline Bojanic et al & C & 3.3 & $24(17-32)$ & $(14-20)$ & $x x$ & $\mathrm{xx}$ & $\mathrm{xx}$ & 0 & 1 & $x x$ \\
\hline Burne et al & C & $4.8(1-15)$ & $n=6$ & $x x$ & $x x$ & 208 (57-796) & $3 / 11$ at 4 years & $x x$ & 4 & $x x$ \\
\hline \multirow[t]{3}{*}{ Khan et al } & $\mathrm{C}$ and $\mathrm{S}$ & 4 ( 3 days -5 years) & & $x x$ & $x x$ & $\mathrm{xx}$ & $\mathrm{xx}$ & & $x x$ & $x x$ \\
\hline & s & & 15.2 & & & & & 1 & & \\
\hline & C & & 21 & & & & & 39 & & \\
\hline McCormick et al & $S$ & 4.3 & $x x$ & $x x$ & $x x$ & $x x$ & $x x$ & 2 & $x x$ & $1 \mathrm{SI}$ \\
\hline \multirow[t]{3}{*}{ Saxena et al ${ }^{19}$} & $\mathrm{C}$ and $\mathrm{S}$ & 5.0 (1 day -4 years) & & $x x$ & $x x$ & $x x$ & $x x$ & $x x$ & & \\
\hline & s & & $12.4(6-20)^{*}$ & & & & & & & $1 \mathrm{HWR}$ \\
\hline & C & & $17.2(12-52)$ & & & & & & 5 & \\
\hline \multirow[t]{3}{*}{ Saxena and Fullem ${ }^{36}$} & $\mathrm{C}$ and $\mathrm{S}$ & & $t$ & $x x$ & $x x$ & $x x$ & $x x$ & & $x x$ & 0 \\
\hline & s & $25.5(3-240) \ddagger$ & $16.4 \pm 4.8$ & & & & & 0 & 0 & $1 \mathrm{HWR}$ \\
\hline & C & $2.25(0.5-6)$ & $14.8 \pm 6.4$ & & & & & 0 & 0 & \\
\hline \multirow[t]{3}{*}{ Torg et al } & $\mathrm{C}$ and $\mathrm{S}$ & $7.2(0-38)$ & & $x x$ & $x x$ & $x x$ & $x x$ & & & \\
\hline & S & & $28(26-30)$ & $x x$ & $x x$ & $x x$ & $x x$ & 0 & 0 & 0 \\
\hline & C & & $21(11-48)$ & $x x$ & $x x$ & $x x$ & $x x$ & $1(1)$ & 3 & $x x$ \\
\hline \multicolumn{11}{|l|}{ Fifth metatarsal } \\
\hline \multirow[t]{3}{*}{ Chuckpaiwong et al } & $\mathrm{C}$ and $\mathrm{S}$ & $x x$ & & $x x$ & & $x x$ & & 2 & 0 & $3 \mathrm{HWR}$ \\
\hline & $\mathrm{s}$ & & $15.2 \pm 10.5$ & & $3.2(2.2)$ & & $39.2(87.6)$ & 0 & & \\
\hline & C & & $26.3 \pm 11.0$ & & $2.5(0.8)$ & & $19.2(21.6)$ & 1 & & \\
\hline DeLee et al & s & $(3.0-18.0)$ & $8.5(7-14)$ & $x x$ & $\mathrm{xx}$ & $4.5(3-6)$ & $7.5(6-8)$ & 0 & 0 & 0 \\
\hline \multirow[t]{3}{*}{ Ekstrand et al } & $\mathrm{C}$ and $\mathrm{S}$ & $\mathrm{xx}$ & & $x x$ & is RT sport & is RT sport & $\mathrm{xx}$ & & & $x x$ \\
\hline & $S$ & & $12(6.1-17.6)$ & & & & & 0 & 5 & \\
\hline & C & & $9.9(8.4-11.1)$ & & & & & 1 & 3 & \\
\hline Josefsson et al & $c$ & $0.07(0-0.7)$ & All & $x x$ & $x x$ & All at final FU & 13 at final FU & 0 & 2 & $x x$ \\
\hline Lee et al & s & $\mathrm{xx}$ & $14.1(5.7-30.3) \S$ & $\mathrm{xx}$ & $x x$ & $\mathrm{xx}$ & is RT sport & 99 & $6^{* *}$ & $x x$ \\
\hline Nagao et al & s & $x x$ & $11.2(6-25)$ & $6.3(3-12.7)$ & $x x$ & $\mathrm{xx}$ & $8.8(4-20.1)$ & $2(1)$ & 0 & 0 \\
\hline Pecina et al & s & $x x$ & $9(5-14)$ & $\mathrm{xx}$ & $x x$ & All at final FU & $\mathrm{xx}$ & $x x$ & 1 & 0 \\
\hline \multirow[t]{3}{*}{ Popovic et al } & s & & & $x x$ & $x x$ & & & $x x$ & & $x x$ \\
\hline & s & $0.7(0.5-0.8)$ & $12.2(11-17)$ & & & $6(5-8)$ & $11.3(9-11.8)$ & & 3 & \\
\hline & s & $1(0.8-1.3)$ & $12(12-12)$ & & & $7.1(6-9.2)$ & $10.1(8.6-10.5)$ & & 0 & \\
\hline 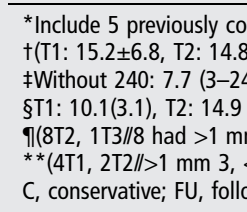 & $\begin{array}{l}\text { rvative treated } \\
\text { 2, T3: } 16.8 \pm 0.3 \\
\\
\text { 3), T3: } 17.6 \text { (7.4 } \\
\text { lantair gap). } \\
\text { nm 3). } \\
\text { up; HWR, hardn }\end{array}$ & $\begin{array}{l}\mathrm{p}<1 \mathrm{~mm} 10.1 \text { (4.3), >1 m } \\
\text { removal; RT, return to; } \mathrm{S}, \mathrm{s}\end{array}$ & $\begin{array}{l}8.1 \text { (7.4). } \\
\text { ery; SI, superficial ir }\end{array}$ & & & & & & & \\
\hline
\end{tabular}




\begin{tabular}{lll} 
Table 3 & Fracture classifications \\
\hline Classification & $\begin{array}{l}\text { Navicular } \\
\text { (Saxena } \text { et } \text { al }^{19} \text { ) }\end{array}$ & Fifth metatarsal (Torg) \\
\hline T1 & $\begin{array}{l}\text { Dorsal cortical } \\
\text { fracture }\end{array}$ & $\begin{array}{l}\text { No intramedullary sclerosis, a fracture line } \\
\text { with sharp margins, no widening or } \\
\text { radiolucency, minimum cortical } \\
\text { hypertrophy or evidence of periosteal } \\
\text { reaction }\end{array}$ \\
T2 & $\begin{array}{l}\text { Dorsal fracture into } \\
\text { navicular body }\end{array}$ & $\begin{array}{l}\text { Fracture line involving both cortices, } \\
\text { associated periosteal new bone; a } \\
\text { widened fracture line with adjacent } \\
\text { radiolucency and evidence of } \\
\text { intramedullary sclerosis }\end{array}$ \\
T3 & $\begin{array}{l}\text { Wide fracture line with periosteal new } \\
\text { bone and radiolucency, complete } \\
\text { obliteration of the medullary canal at the } \\
\text { fracture site by sclerotic bone }\end{array}$ \\
second cortex
\end{tabular}

Radiological fracture healing was mainly evaluated by using radiographs. Mean time of healing in the surgical group ranged from $7.5^{20}$ to 39.2 weeks. $^{40}$ In the conservative group, this was 19.2 weeks based on one small study. ${ }^{40}$ Surgically treated patients had a non-union rate of $4.6 \%$ and a refracture rate of $6.9 \%$. No infections or wound complications were reported. Conservatively treated patients had a non-union rate of $7.1 \%$ and a refracture rate of $17.9 \%$.

No economic data were found for any of the fracture locations. Overall, the evidence was scored as 'very low quality'.

\section{DISCUSSION}

The main goal of the present study was to compare the time to return to sport, activity and work after conservative or operative treatment of stress fractures of the anterior tibial cortex, the navicular bone and the fifth metatarsal. We found very lowquality evidence only and it remains unclear whether surgery is more effective than conservative treatment for high-risk stress fractures of the lower leg. The key issues depicted by this review are the small number of studies, the small sample sizes and the high RoB of the studies. Also, there were only two cohorts of which only one included both surgery and conservative treatment. According to the GRADE approach, the level of evidence is of very low quality, suggesting that there is great uncertainty about the effect estimate. It would be inappropriate to draw firm conclusions about a possible superior therapy.

The methodology applied to the search and selection of articles for inclusion is one of the strengths of this review. All major databases were systematically searched without restrictions in language. To minimise outcome bias, we only included studies with a consecutive series of 10 or more patients. For the first time in stress fracture reviews, adequate quality assessment of included studies was performed to reveal the methodological weakness of current literature on which we base our treatment policies.

Several reviews already reported advice on best treatment, ${ }^{27} 2845$ even though little and poor evidence on conservative and surgical methods was available. Importantly, we found no RCTs and no direct comparison between surgical or conservative treatment; hence, the quality of evidence was very low. Furthermore, as stated in a comment on the navicular stress fracture review by Torg et al, ${ }^{46}$ there is a lack of focus on different fracture types (table 3). This statement might be applicable to all three high demand stress fractures, since it is likely that outcomes differ with severity of the fracture.
No studies collected economic data; therefore, the cost or cost-effectiveness of either conservative or surgical treatment is unknown.

\section{Anterior tibial cortex}

The lack of evidence for initial surgery refrain us from comparing surgery and conservative treatment. The few results for conservative treatment provide worrying outcomes since most patients did not return to their previous level of sport and a high rate of non-unions was found. Clinicians must keep these results in mind and explain the difficulties in anterior tibial stress fractures to their patients. Extensive follow-up of conservatively treated is advised to assess proper healing and to intervene if non-union occurs.

Small case series not included in this review, which studied (secondary) tension band plating, report interesting time to return to sport of approximately 3 months. This was achieved without major complications in union or infection. ${ }^{47} 48$ However, further evaluation of surgical results in larger studies is needed.

Creating awareness of this condition and a better diagnostic approach potentially reduces the time between the onset of symptoms until start of treatment, as this was reported to be quite extreme in most cases. ${ }^{13} 15$

\section{Navicular}

Significant differences could not be calculated; however, current data suggest that surgical treatment results in an earlier return to sport (16.4 vs 21.7 weeks). Postoperative complications were rarely reported (table 2). Among conservatively treated patients, the complication rates were high, especially delayed unions and refractures. A NWBC treatment of at least 6 weeks results in less treatment failures than conservative treatment without non-WB immobilisation. ${ }^{38}$ Therefore, this would be the conservative treatment of choice. The surgical methods were different in reviewed studies, all using screws for internal fixation and in some with additional bone graft. Unfortunately, data are insufficient to depict the best surgical treatment.

Owing to the lack of RCTs and non-randomised trials (comparison studies), we could not perform a statistical comparison between pooled surgery and conservative outcomes. This is in contradiction to the review by Torg et al, ${ }^{28}$ where a comparative meta-analysis was performed. Their review also included case reports, thus limiting the level of evidence provided. This could have introduced a considerable RoB, which was not assessed. Some studies included very few cases, but in the performed meta-analysis, data were not weighted according to study size. In addition, the already low numbers of surgical cases included in their analysis were secondary treatments due to delayed union or non-union after conservative treatment. More importantly, no significant difference between surgical treatment and NWBC was found.

With these limitations in mind, appropriate advice for standard of care cannot be made.

\section{Proximal fifth metatarsal}

Patients with this type of fracture must account for time away from sports of approximately 14 weeks. Significant differences could not be calculated; however, surgical treatment resulted in an earlier return to sport (13.8 vs 19.2 weeks). Union problems and refractures seem less prevalent after surgical treatment. Conservative treatment details were limited, consisting of WB and non-WB protocols. Unfortunately, evidence is still lacking to advise on one adequate method. Operative treatment varied, but is mostly based on providing compression by means of an 
intramedullary screw fixation or tension band wiring. Even though sclerotic aspects of the fracture are seen on radiographs (eg, Torg 3 fractures), it is not mentioned that surfaces are revitalised prior to compression. This is a known procedure in fracture non-union surgery and might be of additional value.

Furthermore, the distinction and interpretation of stress fractures or Jones' fractures can be difficult; therefore verification by contacting the authors was needed to ensure inclusion of stress fractures only and reduce possible selection bias. Though some claim this differentiation is not necessary ${ }^{40}$ Kerkhoffs et a ${ }^{27}$ showed a difference in outcomes between these fracture types. The same review advised surgical treatment in high-level athletes, but evidence was restrained from RoB assessment, which appears to be considerable. Roche $e t a l^{49}$ reviewed proximal metatarsal fractures as well; however, the outcomes of both stress and acute fractures were interpreted as one group and are therefore different from the current study.

All included studies had a high RoB and the evidence was of very low quality. Future studies should include larger sample sizes and use more valid study designs. Although it might be difficult to perform randomised controlled trials because of the small number of eligible patients and their professional athletic background, at least a prospective study design should be used including all patients and with time to return to activity, work and sport as main outcomes. Complication rates of high-risk stress fractures are high, especially for the anterior tibia. To monitor outcomes and complications, follow-up needs to be long enough to measure these outcomes. At present, it remains unclear whether surgery is more effective than conservative treatment for high-risk stress fractures of the lower leg.

Acknowledgements The authors would like to thank Faridi van EttenJamaludin, clinical librarian, their hospital (blinded for peer review), for constructing an adequate and extensive search strategy for all databases.

Contributors WHM wrote protocol, registered protocol, performed search, reviewed eligible articles, extracted data, wrote manuscript and submitted manuscript. HW reviewed eligible articles, extracted data, performed data analysis and wrote manuscript. CNvD supported and corrected manuscript. MWvT performed data analysis, supported and corrected manuscript. GMK was involved in protocol suggestion, supported and corrected protocol and manuscript. C-WCL wrote protocol, performed search strategy, was third reviewer in case of disagreement, supported and corrected manuscript.

\section{What are the new findings?}

- High-risk stress fractures of the lower leg are prone to be a career ending injury in athletes.

- Conservative therapy in anterior tibial stress fractures reported very low success rates and only a few athletes returned to their previous sports level.

- Navicular and fifth metatarsal stress fractures seem to have an earlier return to sport after surgical therapy.

- Prospective research with clear premade definitions on return to sport and time to fracture healing is needed to disclose firm conclusions about treatment of high-risk stress fractures.

Competing interests WHM is funded by a PhD Scholarship provided by the Academic Medical Center of Amsterdam, The Netherlands. HW is sponsored by a Marti Keunig Eckhardt Foundation Grant.

Provenance and peer review Not commissioned; externally peer reviewed.

\section{REFERENCES}

1 Rome K, Handoll Helen HG, Ashford R, et al. Interventions for preventing and treating stress fractures and stress reactions of bone of the lower limbs in young adults. Cochrane Database Syst Rev 2005;(2):CD000450. http://onlinelibrary.wiley. com/doi/10.1002/14651858.CD000450.pub2/abstract

2 Armstrong IDW, Rue JPH, Wilckens JH, et al. Stress fracture injury in young military men and women. Bone 2004;35:806-16.

3 Kelly AKW, Hame SL. Managing stress fractures in athletes. J Musculoskelet Med 2005;22:463.

4 Shindle MK, Endo Y, Warren RF, et al. Stress fractures about the tibia, foot, and ankle. J Am Acad Orthop Surg 2012;20:167-76.

5 Rolf $\mathrm{C}$, Ekenman I, Tornqvist $\mathrm{H}$, et al. The anterior stress fracture of the tibia: an atrophic pseudoarthosis? Scand J Med Sci Sports 1997;7:249-52.

6 Torg JS, Pavlov H, Cooley LH, et al. Stress fractures of the tarsal navicular. A retrospective review of twenty-one cases. J Bone Joint Surg Am 1982;64:700-12.

7 Shereff MJ, Yang QM, Kummer FJ, et al. Vascular anatomy of the fifth metatarsal. Foot Ankle 1991;11:350-3.

8 Smith JW, Arnoczky SP, Hersh A. The intraosseous blood supply of the fifth metatarsal: implications for proximal fracture healing. Foot Ankle 1992;13:143-52.

9 McKeon KE, Johnson JE, McCormick JJ, et al. The intraosseous and extraosseous vascular supply of the fifth metatarsal: implications for fifth metatarsal osteotomy. Foot Ankle Int 2013;34:117-23.

10 Boden BP, Osbahr DC. High-risk stress fractures: evaluation and treatment. J Am Acad Orthop Surg 2000;8:344-53.

11 Brukner P, Bennell K. Stress fractures in female athletes. Diagn Manag Rehabil Sports Med 1997;24:419-29.

12 Kaeding CC, Yu JR, Wright R, et al. Management and return to play of stress fractures. Clin J Sport Med 2005;15:442-7.

13 Orava S, Hulkko A. Stress fracture of the mid-tibial shaft. Acta Orthop Scand 1984;55:35-7.

14 Liimatainen E, Sarimo J, Hulkko A, et al. Anterior mid-tibial stress fractures. Results of surgical treatment. Scand J Surg 2009:98:244-9.

15 Johansson C, Ekenman I, Lewander R. Stress fracture of the tibia in athletes: diagnosis and natural course. Scand J Med Sci Sports 1992;2:87-91.

16 Plasschaert VF, Johansson CG, Micheli LJ. Anterior tibial stress fracture treated with intramedullary nailing: a case report. Clin J Sport Med 1995;5:58-61; discussion-2.

17 Mann JA, Pedowitz DI. Evaluation and treatment of navicular stress fractures, including nonunions, revision surgery, and persistent pain after treatment. Foot Ankle Clin 2009;14:187-204.

18 Snyder RA, Koester MC, Dunn WR. Epidemiology of stress fractures. Clin Sports Med 2006;25:37-52.

19 Saxena A, Fullem B, Hannaford D. Results of treatment of 22 navicular stress fractures and a new proposed radiographic classification system. J Foot Ankle Surg 2000;39:96-103

20 DeLee JC, Evans JP, Julian J. Stress fracture of the fifth metatarsal. Am J Sports Med 1983;11:349-53.

21 Ekstrand J, van Dijk CN. Fifth metatarsal fractures among male professional footballers: a potential career-ending disease. Br J Sports Med 2013;47:754-8.

22 Hens J, Martens M. Surgical treatment of Jones fractures. Arch Orthop Trauma Surg 1990;109:277-9.

23 Lee KT, Kim KC, Park YU, et al. Radiographic evaluation of foot structure following fifth metatarsal stress fracture. Foot Ankle Int 2011;32:796-801.

24 Torg JS, Balduini FC, Zelko RR, et al. Fractures of the base of the fifth metatarsal distal to the tuberosity. Classification and guidelines for non-surgical and surgical management. J Bone Joint Surg Am 1984;66:209-14.

25 Lawrence SJ, Botte MJ. Jones' fractures and related fractures of the proximal fifth metatarsal. Foot Ankle 1993;14:358-65.

26 Lee KT, Park YU, Young KW, et al. The plantar gap: another prognostic factor for fifth metatarsal stress fracture. Am J Sports Med 2011;39:2206-11.

27 Kerkhoffs GM, Versteegh VE, Sierevelt IN, et al. Treatment of proximal metatarsal V fractures in athletes and non-athletes. Br J Sports Med 2012;46:644-8.

28 Torg JS, Moyer J, Gaughan JP, et al. Management of tarsal navicular stress fractures: conservative versus surgical treatment: a meta-analysis. Am J Sports Med 2010;38:1048-53.

29 Moher D, Liberati A, Tetzlaff J, et al. Preferred reporting items for systematic reviews and meta-analyses: the PRISMA statement. BMJ 2009;339:b2535.

30 Higgins JPT, Altman DG, Sterne JAC. Chapter 8: assessing risk of bias in included studies. Cochrane Handbook for Systematic Reviews of Interventions. The Cochrane Collaboration, 2011.

31 Guyatt GH, Oxman AD, Vist G, et al. GRADE guidelines: 4. Rating the quality of evidence-study limitations (risk of bias). J Clin Epidemiol 2011;64:407-15.

32 Guyatt $G H$, Oxman $A D$, Vist $G E$, et al. GRADE: an emerging consensus on rating quality of evidence and strength of recommendations. BMJ 2008;336:924-6.

33 Benazzo F, Mosconi M, Beccarisi G, et al. Use of capacitive coupled electric fields in stress fractures in athletes. Clin Orthop Relat Res 1995;310:145-9.

34 Bojanic I, Pecina MM. [Conservative treatment of stress fractures of the tarsal navicular in athletes]. Rev Chir Orthop Reparatrice Appar Mot 1997;83:133-8. 
35 McCormick JJ, Bray CC, Davis WH, et al. Clinical and computed tomography evaluation of surgical outcomes in tarsal navicular stress fractures. Am J Sports Med 2011;39:1741-8.

36 Saxena A, Fullem B. Navicular stress fractures: a prospective study on athletes. Foot Ankle Int 2006;27:917-21.

37 Burne SG, Mahoney CM, Forster BB, et al. Tarsal navicular stress injury: long-term outcome and clinicoradiological correlation using both computed tomography and magnetic resonance imaging. Am J Sports Med 2005;33:1875-81.

38 Khan KM, Fuller PJ, Brukner PD, et al. Outcome of conservative and surgical management of navicular stress fracture in athletes. Eighty-six cases proven with computerized tomography. Am J Sports Med 1992;20:657-66.

39 Orava S, Karpakka J, Hulkko A, et al. Diagnosis and treatment of stress fractures located at the mid-tibial shaft in athletes. Int J Sports Med 1991;12:419-22.

40 Chuckpaiwong B, Queen RM, Easley ME, et al. Distinguishing Jones and proximal diaphyseal fractures of the fifth metatarsal. Clin Orthop Relat Res 2008;466:1966-70.

41 Josefsson PO, Karlsson M, Redlund-Johnell I, et al. Closed treatment of Jones fracture. Good results in 40 cases after 11-26 years. Acta Orthop Scand 1994;65:545-7.

42 Nagao M, Saita Y, Kameda S, et al. Headless compression screw fixation of jones fractures: an outcomes study in Japanese athletes. Am J Sports Med 2012;40:2578-82.
43 Pecina M, Bojanic I, Smoljanovic T, et al. Surgical treatment of diaphyseal stress fractures of the fifth metatarsal in competitive athletes: long-term follow-up and computerized pedobarographic analysis. J Am Podiatr Med Assoc 2011;101:517-22.

44 Popovic N, Jalali A, Georis P, et al. Proximal fifth metatarsal diaphyseal stress fracture in football players. Foot Ankle Surg 2005;11:135-41.

45 Thevendran G, Deol RS, Calder JD. Fifth metatarsal fractures in the athlete: evidence for management. Foot Ankle Clin 2013;18:237-54.

46 Saxena A, Fullem B. Comment on Torg et al, "management of tarsal navicular stress fractures: conservative versus surgical treatment". Am J Sports Med 2010;38: NP3-5; author reply NP.

47 Cruz AS, de Hollanda JP, Junior AD, et al. Anterior tibial stress fractures treated with anterior tension band plating in high-performance athletes. Knee Surg Sports Traumatol Arthrosc 2013;21:1447-50.

48 Borens 0, Sen MK, Huang RC, et al. Anterior tension band plating for anterior tibial stress fractures in high-performance female athletes: a report of 4 cases. J Orthop Trauma 2006;20:425-30.

49 Roche AJ, Calder JD. Treatment and return to sport following a Jones fracture of the fifth metatarsal: a systematic review. Knee Surg Sports Traumatol Arthrosc 2013;21:1307-15. 